DOI 10. 18307/2016. 0405

(C) 2016 by Journal of Lake Sciences

\title{
生物沸石薄层覆盖削减富营养化水体磷负荷”
}

\author{
周真明 ${ }^{1,2}$, 黄廷林 ${ }^{2 * *}$, 苑宝玲 ${ }^{1}$ \\ (1:华侨大学土木工程学院,厦门 361021) \\ (2:西安建筑科技大学环境与市政工程学院,西安 710055)
}

\begin{abstract}
摘 要: 以江苏扬州古运河富营养化水体为对象, 现场围隔实验研究生物沸石薄层覆盖削减富营养化水体磷负荷可行 性, 考察生物沸石覆盖削减上覆水、底泥间隙水和底泥中不同形态磷的削减效果, 讨论生物沸石覆盖修复过程中不同形 态磷转化机制. 结果表明, 覆盖强度为 $2 \mathrm{~kg} / \mathrm{m}^{2}$ 的生物沸石覆盖 (厚度约 $2 \mathrm{~mm}$ ) 对上覆水中总磷的削减率为 $57.41 \%$, 对上 覆水中正磷酸盐的削减率为 $60.03 \%$; 对底泥间隙水中正磷酸盐的削减率为 $59.80 \%$; 对表层底泥 $(0 \sim 20 \mathrm{~cm})$ 中总磷削减 率为 $11.28 \%$, 对无机磷削减率为 $11.82 \%$, 对有机磷削减率为 $11.11 \%$. 生物沸石覆盖能将底泥中不稳定的无机磷 (可溶性 磷、铁结合态磷、铝结合态磷) 或少部分较稳定的无机磷 (钲结合态磷) 转化为稳定的无机磷 (包裹磷), 说明生物沸石覆 盖不仅能削减液相中磷负荷, 而且能将固相中不稳定的无机磷转化为稳定的无机磷; 可见, 生物沸石薄层覆盖能有效削 减富营养化水体磷负荷, 利用生物沸石薄层覆盖削减富营养化水体磷负荷是可行的, 但需要进一步研究富营养化水体底 泥生物薄层覆盖修复过程中不同形态无机磷转化机制.
\end{abstract}

关键词: 底泥;覆盖;磷;生物沸石;富营养化水体;扬州古运河

\section{Biozeolite thin-layer capping for reducing the phosphorus load in eutrophic water body}

\section{ZHOU Zhenming ${ }^{1,2}$, HUANG Tinglin ${ }^{2 * *}$ \& YUAN Baoling}

(1: College of Civil Engineering, Huaqiao University, Xiamen 361021, P.R. China)

(2: School of Environmental and Municipal Engineering, Xi'an University of Architecture \& Technology, Xi'an 710055, P.R. China)

\begin{abstract}
The feasibility of reducing phosphorus load in eutrophic water body using biozeolite thin-layer capping was examined through a field incubation experiment in Ancient Canal in Yangzhou, Jiangsu Province, China. The reduction efficiency of different phosphorus in overlying water, interstitial water and sediments was estimated. The mechanisms of different phosphorus transportation and transformation in remediation process of sediment using biozeolite thin-layer capping were discussed. The results showed that the reduction efficiency of total phosphorus and orthophosphate in overlying water by biozeolite thin-layer capping of dose rate of $2 \mathrm{~kg} / \mathrm{m}^{2}$ ( the thick of $2 \mathrm{~mm}$ ) were $57.41 \%$ and $60.03 \%$, respectively. The orthophosphate reduction efficiency in interstitial water from sediments was $59.80 \%$. The reduction efficiencies of total phosphorus, inorganic phosphorus and organic phosphorus in surface sediments $(0-20 \mathrm{~cm})$ were $11.28 \%, 11.82 \%$ and $11.11 \%$, respectively. Unsteady inorganic phosphorus (e.g. dissolved phosphorus, iron bound phosphorus, aluminium bound phosphorus) and relatively steady inorganic phosphorus (e.g. calcium bound phosphorus) were converted into very steady inorganic phosphorus (e.g. residual phosphorus) using biozeolite thin-layer capping, indicating that biozeolite thin-layer capping could not only reduce phosphorus load in liquid, but also convert unsteady inorganic phosphorus into very steady inorganic phosphorus. Therefore, biozeolite thin-layer capping can reduce phosphorus load in eutrophic water body efficiently, and phosphorus load reduction in eutrophic water body using biozeolite thin-layer capping is feasible. However, it is urgent to understand the mechanisms of different inorganic phosphorus transportation and transformation in remediation process of sediment using biozeolite thin-layer capping.
\end{abstract}

* 国家水体污染控制与治理科技重大专项(2009ZX07317-007-1-2)、国家自然科学基金项目(51408243)、福建省自然 科学基金项目(2015J01213) 和华侨大学科研基金项目(14BS216) 联合资助. 2015-04-15 收稿; 2015-10-26 收 修改稿. 周真明( 1981 ), 男, 博士, 讲师;E-mail : zhenming@ hqu.edu.cn.

** 通信作者;E-mail: huangtinglin@ xauat.edu.cn. 
Keywords: Sediment; capping; phosphorus; biozeolite; eutrophic water body; Yangzhou Ancient Canal

水体富营养化是当今世界水污染防治面临的重要难题之一. 随着我国工业化和城市化的发展, 水体富 营养化趋于严重. 氮和磷是水体富营养化的主要限制因子, 其中磷被普遍认为是关键限制因子, 控制水体氮 和磷浓度能有效抑制水体富营养化 ${ }^{[1]}$. 底泥是水体氮和磷的“源” 和“汇”, 底泥氮磷释放是水体氮磷主要来 源之一 ${ }^{[2-3]}$, 因此, 削减底泥氮磷释放能有效控制水体富营养化. 底泥原位覆盖法是当今国内外学者研究热 点, 并在欧美、日本等国得到广泛应用 ${ }^{[4]}$. 底泥覆盖材料从传统沙子 ${ }^{[5]}$ 等发展到现今铁盐 ${ }^{[6]}$ 、铝盐 ${ }^{[7]}$ 、钻盐

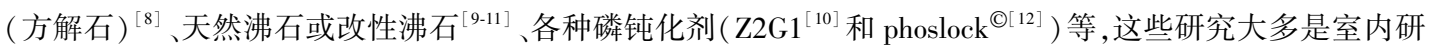
究, 且主要集中在对上覆水磷的控制效果及机制研究, 对底泥和间隙水不同形态磷削减效果的现场原位实 验研究鲜见报道.

笔者前期所在课题组自主研发了污染底泥生物活性覆盖材料 (生物沸石), 通过实验室静态模拟和现场 原位实验研究以及示范工程应用 ${ }^{[11,13]}$, 结果表明: 生物沸石薄层覆盖不仅能有效削减上覆水的氮负荷, 而且 也削减了底泥中的氮负荷, 同时高效菌生物作用能实现沸石吸附氨氮能力的原位再生. 为了考察生物沸石 覆盖是否具备同步削减水体氮和磷功能, 更有效地控制水体富营养化, 本文以扬州古运河富营养化水体为 研究对象, 通过现场围隔实验研究了生物沸石薄层覆盖削减富营养化水体磷负荷可行性, 考察生物沸石覆 盖对上覆水体总磷 $(\mathrm{TP})$ 和正磷酸盐 $\left(\mathrm{PO}_{4}^{3-}-\mathrm{P}\right)$ 削减效果、对底泥间隙水中 $\mathrm{PO}_{4}^{3-}-\mathrm{P}$ 削减效果以及对底泥不同 形态磷削减效果, 讨论生物沸石覆盖削减磷负荷作用机理. 研究结果可为生物沸石薄层覆盖技术控制水体 富营养化的实际应用提供理论依据和技术支撑.

\section{1 材料与方法}

\section{1 实验场地及装置}

现场围隔实验场地在古运河扬州城区三湾段 (图 1). 古运 河扬州段是京杭大运河最古老的一段,其中古运河扬州城区段 从湾头到瓜洲, 全长约 $30 \mathrm{~km}$, 河宽 $24 \mathrm{~m}$, 水深 3 5 m. 目前, 古 运河扬州市区段已经禁止通航,其功能主要是泄洪;该段大部分 时间水体流动缓慢,趋于静止 (除掉从京杭大运河引水到古运 河期间); 该段水体富营养化严重, 时而出现藻类暴发, 主要污 染源是城市生活污水、工业、农业面源污染.

现场围隔的实验装置是塑料管, 管长 $6 \mathrm{~m}$ 、直径 $1000 \mathrm{~mm}$, 材料是硬聚氯乙烯 ( U-PVC), 类型是双壁波纹管, 管壁截面为 双层结构,其内壁光滑平整,外壁等距排列的具有梯形中空结构 的管材. 围隔施工过程是: 通过吊车将塑料管垂直轻轻放人河 中, 开始依靠塑料管自身重力在底泥中缓慢下沉, 当达到稳定 时, 采用吊车长臂轻压塑料管, 使其稳定地插人底泥, 塑料管高 出河水常水位 $0.5 \mathrm{~m}$, 其余的长度切掉. 现场围隔实验参数: 塑 料管插人泥深 $2.5 \mathrm{~m}$ 左右, 管内上覆水深 $2.0 \mathrm{~m}$ 左右, 上覆水体 积约 $1.57 \mathrm{~m}^{3}$; 围隔实验共有 2 根塑料管, $1^{\#}$ 为对照系统 (无覆 盖) $; 2^{\#}$ 为生物沸石覆盖系统 (生物沸石覆盖强度为 $2 \mathrm{~kg} / \mathrm{m}^{2}$, 覆 盖厚度约为 $2 \mathrm{~mm}$ ).

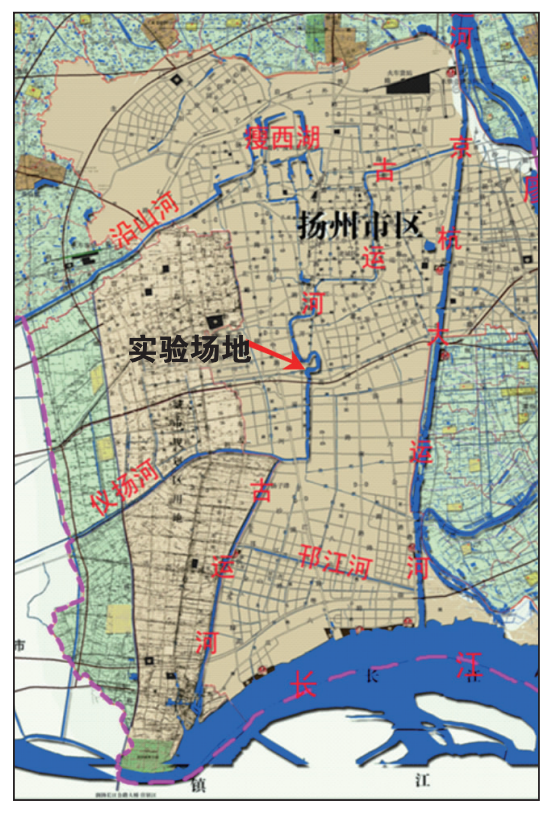

图 1 扬州古运河现场围隔实验场地位置

Fig. 1 Location of the field for enclosure experiment in Yangzhou Ancient Canal

\section{2 实验材料}

实验所用的沸石为中国内蒙巴彦淖尔的天然沸石, 经过 X 射线衍射仪 (XRD) 检测, 其主要矿物成分为 斜发沸石, 伴生矿为正长石和石英. 沸石表观颜色为红褐色, 粒径为 $1 \sim 2 \mathrm{~mm}$, 比表面积为 $42.51 \mathrm{~m}^{2} / \mathrm{g}$, 孔隙 率为 $36.55 \%$, 平均孔径为 $6.75 \mathrm{~nm}$, 堆积密度为 $1.01 \mathrm{~g} / \mathrm{cm}^{3}$, 真实密度为 $2.29 \mathrm{~g} / \mathrm{cm}^{3}$. 经过 $X$ 射线苂光光谱仪 (XRF) 测定,该沸石的主要化学元素成分为:Si 73.98\% ; Al 8.99\%;Ca 5.07\%;K 4.68\%;Fe 3.11\% ; Na 2.13\%; 
$\mathrm{Mg} 1.51 \%$; 还含有 $\mathrm{Mn} 、 \mathrm{Ti} 、 \mathrm{Zn} 、 \mathrm{Cu} 、 \mathrm{Ni} 、 \mathrm{Co} 、 \mathrm{Sr} 、 \mathrm{Zr} 、 \mathrm{Rb}$ 等其它微量元素, 合计 $0.53 \% ; \mathrm{Si} / \mathrm{Al}=8.23$.

实验所用的生物沸石制备过程如下: (1) 从污染底泥中分离、笁选出高效菌, 本课题组获得 4 株高效菌, 其中 2 株高效异养硝化细菌 (均为芽孢杆菌属, Bacillus sp.) 和 2 株高效好氧反硝化菌 (均为不动杆菌属, Acinetobacter sp.),4 株菌的生理生化特征详见参考文献 $[11,13]$; (2) 将 4 株高效菌液按照等体积比例混合 制备混合菌液, 再将混合菌液与灭菌的古运河原水按照 1 :9体积比混合制备混合液; (3) 将天然沸石浸置在 混合液中, 混合液的液面高于沸石层 $5 \mathrm{~cm}$ 左右, 通过连续曝气挂膜法制备生物沸石, 制备过程中混合液的 DO $>1 \mathrm{mg} / \mathrm{L}$. 具体详细制备过程参考文献 $[11,13]$.

现场围隔实验装置完成后, 实验系统中上覆水水质如表 1 所示. 由于进行现场围隔时, 很难做到每次对 底泥扰动程度相同, 因此造成对照系统和生物沸石覆盖系统中水质有些差异.

表 1 扬州古运河上覆水水质

Tab.1 Water quality of overlying water in experimental systems

\begin{tabular}{lcccccccc}
\hline 实验系统 & $\mathrm{pH}$ 值 & $\begin{array}{c}\mathrm{COD}_{\mathrm{Cr}} / \\
(\mathrm{mg} / \mathrm{L})\end{array}$ & $\begin{array}{c}\mathrm{TN} / \\
(\mathrm{mg} / \mathrm{L})\end{array}$ & $\begin{array}{c}\mathrm{NH}_{4}^{+}-\mathrm{N} / \\
(\mathrm{mg} / \mathrm{L})\end{array}$ & $\begin{array}{c}\mathrm{NO}_{2}^{-}-\mathrm{N} / \\
(\mathrm{mg} / \mathrm{L})\end{array}$ & $\begin{array}{c}\mathrm{NO}_{3}^{-}-\mathrm{N} / \\
(\mathrm{mg} / \mathrm{L})\end{array}$ & $\begin{array}{c}\mathrm{TP} / \\
(\mathrm{mg} / \mathrm{L})\end{array}$ & $\begin{array}{c}\mathrm{PO}_{4}^{3-}-\mathrm{P} / \\
(\mathrm{mg} / \mathrm{L})\end{array}$ \\
\hline 对照 & 7.41 & 39.6 & 5.31 & 4.23 & 0.039 & 0.18 & 0.17 & 0.10 \\
生物沸石 & 7.47 & 41.2 & 5.42 & 4.05 & 0.059 & 0.21 & 0.14 & 0.085 \\
\hline
\end{tabular}

\section{3 实验方法}

在现场围隔前, 先从河中采用自制柱状取泥装置取柱状泥分析不同形态磷含量, 作为实验系统泥中不 同形态磷含量初始值. 现场围隔的实验装置在 2011 年 4 月 21 日搭建完成, 实验于 22 日开始运行, 第 $74 \mathrm{~d}(7$ 月 5 日) 开始, 扬州连续几天暴雨, 古运河水位暴涨, 第 $78 \mathrm{~d}$, 现场围隔实验系统被淹没, 致使实验系统的上 覆水与古运河水体进行自然交换, 第 $79 \mathrm{~d}$ 一早, 古运河水位下降, 因此, 本文实验系统上覆水 $\mathrm{TP}$ 和 $\mathrm{PO}_{4}^{3-}-\mathrm{P}$ 监测到 $73 \mathrm{~d}$ 就结束, 但为了考察生物沸石修复过程中底泥中不同磷迁移转化机制, 现场围隔实验系统继续 保留,在第 $121 \mathrm{~d}$ 时, 从实验系统取出柱状泥进行分析.

实验期间,每 $2 \mathrm{~d}$ 测定系统中距水面 $0.5 \mathrm{~cm}$ 水深处水温、溶解氧 ( DO) 和 $\mathrm{pH}$ 值; 每 $6 \sim 7 \mathrm{~d}$ 取水样测定 TP 和 $\mathrm{PO}_{4}^{3-}-\mathrm{P}$ 浓度, 每次取 $500 \mathrm{ml}$ 水样, 并及时取古运河中一定量原水加人系统中使液面达到原先的刻度线, 保持上覆水体积恒定. 实验历时 $121 \mathrm{~d}$, 从实验系统中取出柱状泥进行分析, 为了避免取泥过程扰动对系统 影响过大, 将 2 根有机玻璃管套在一起, 内管直径 $3.5 \mathrm{~cm}$, 外管直径 $4.5 \mathrm{~cm}$, 管壁厚 $3 \mathrm{~mm}$, 取泥时同时将 2 根 有机玻璃管缓慢插人底泥中, 然后缓慢将内管取出来, 外管留在系统中, 再用自制的橡皮塞将管中底泥推压 出来, 采用薄不锈钢切片分别按 $0 \sim 3 、 3 \sim 6 、 6 \sim 10 、 10 \sim 20 、 20 \sim 50 \mathrm{~cm}$ 厚度把泥样切下来, 部分底泥通过离心 获得间隙水, 根据测试水样需要体积数将间隙水稀释若干倍后测定 TP 和 $\mathrm{PO}_{4}^{3-}$ - $\mathrm{P}$ 浓度; 部分底泥风干、碾 细、过 100 目尼龙篮后待分析; 底泥测试指标为 TP、无机磷 (IP)、有机磷 (OrP)、可溶性磷 (DP)、铝结合态磷 $(\mathrm{Al}-\mathrm{P})$ 、铁结合态磷 $(\mathrm{Fe}-\mathrm{P})$ 、钙结合态磷 ( Ca-P)、包裹磷 $(\mathrm{RP})$. 水样和底泥样品分析时, 做 3 个平行样, 求得 的平均值作为最终各项指标数据.

\section{4 项目测试方法}

1.4.1 沸石相关指标测定方法 主要矿物成分采用 XRD 分析(德国 Bruker-AXS 公司, 型号 D8 Advance, XRD); 主要元素成分采用 XRF 分析 (美国热电公司,型号 RL Thermo EDXRF Quant's, XRF); 比表面积采用 多点 BET 法测定 (贝士德仪器科技 (北京) 有限公司, 型号 3H-2000PS2, 静态容量法、比表面积及孔结构测定 仪); 孔隙率和孔径分布采用 BJH 法 (园筒孔模型) 测定 (测定仪器名称、生产厂家及型号同比表面积) ; 真实 密度采用 $\mathrm{He}$ 置换, 在真空容量吸附装置中测定.

1.4.2 水体相关指标测定方法 水温和 DO 浓度采用便携式溶解氧测定仪测定 (HACH, 型号 HQ30d); $\mathrm{pH}$ 值 采用便携式 $\mathrm{pH}$ 计测定 (上海精密科学仪器有限公司, 型号 PHB-4) ; 总氮 ( TN) 浓度采用过硫酸钾氧化-紫外 分光光度法测定, 氨氮 $\left(\mathrm{NH}_{3}-\mathrm{N}\right)$ 浓度采用纳氏试剂光度法测定, 亚硝态氮 $\left(\mathrm{NO}_{2}^{-}-\mathrm{N}\right)$ 浓度采用 $\mathrm{N}-(1$-奈基 $)$-乙 二胺光度法测定, 硝态氮 $\left(\mathrm{NO}_{3}^{-}-\mathrm{N}\right)$ 浓度采用紫外分光光度法测定, $\mathrm{TP}$ 和 $\mathrm{PO}_{4}^{3-}-\mathrm{P}$ 浓度采用锄锑抗分光光度法 
测定 ${ }^{[14]}$.

1.4.3 底泥相关项目测试方法 底泥 TP 含量采用高氯酸一硫酸消化一钿锑抗分光光度法测定,不同形态无 机磷 (DP、Al-P、Fe-P、Ca-P 和 RP) 采用连续萃取法测定 ${ }^{[15]}$; 本文中的无机磷为不同形态无机磷之和, 即 IP = $\mathrm{DP}+\mathrm{Al}-\mathrm{P}+\mathrm{Fe}-\mathrm{P}+\mathrm{Ca}-\mathrm{P}+\mathrm{RP}$; 本文有机磷为总磷与无机磷之差.

\section{5 数据处理}

上覆水体和底泥中不同形态磷削减率 $P$ 按照公式 (1) 计算:

$$
P=\frac{\left(C_{\mathrm{NC} i}-C_{\mathrm{NC} 0}\right)-\left(C_{\mathrm{C} i}-C_{\mathrm{C} 0}\right)}{C_{\mathrm{NC} i}-C_{\mathrm{NC} 0}} \times 100 \%
$$

式中, $C_{\mathrm{Co}}$ 和 $C_{\mathrm{C} i}$ 分别为初始和第 $i$ 次取样时生物沸石覆盖系统上覆水或底泥中不同形态磷浓度 $(\mathrm{mg} / \mathrm{L}$ 或 $\mathrm{mg} / \mathrm{kg}) ; C_{\mathrm{NCO}}$ 和 $C_{\mathrm{NC} i}$ 分别为初始和第 $i$ 次取样时对照系统上覆水或底泥中不同形态磷浓度 $(\mathrm{mg} / \mathrm{L}$ 或 $\mathrm{mg} /$ $\mathrm{kg}) ; i$ 为取样次数.

采用方差分析生物沸石覆盖系统与对照系统之间削减磷污染物效果的差异.

\section{2 结果与讨论}

\section{1 削减上覆水体中磷负荷能力}

现场围隔实验运行过程中, 对照系统和生物沸石覆盖系统中水温变化范围为 $17.3 \sim 30.3^{\circ} \mathrm{C}$, 系统中水温 变化较大, 呈现上升趋势; 水温升高, 会促进底泥中磷的释放 ${ }^{[16]}$. 对照系统和生物沸石覆盖系统中 DO 浓度 变化范围为 0.32 6.46 mg/L,生物覆盖系统中 DO 浓度整体来看高于对照系统, 水体 DO 浓度低于 $2 \mathrm{mg} / \mathrm{L}$, 底泥中磷释放强度明显增加 ${ }^{[13]}$. 对照系统和生物沸石覆盖系统中的 $\mathrm{pH}$ 值变化范围为 $7.20 \sim 8.12$, 对照系统 与生物沸石覆盖系统中 $\mathrm{pH}$ 值变化差异不大 (图 2).

覆盖强度为 $2 \mathrm{~kg} / \mathrm{m}^{2}$ 的生物沸石覆盖 (厚度约为 $2 \mathrm{~mm}$ ) 对上覆水体中 TP 的削减率为 $43.92 \% \sim 71.07 \%$, 平均值为 $57.41 \%$; 对上覆水中 $\mathrm{PO}_{4}^{3-}-\mathrm{P}$ 的削减率为 $42.29 \% \sim 85.70 \%$, 平均值为 $60.03 \%$ (图 3) ; 说明生物沸石 覆盖能有效削减上覆水体中磷负荷, 利用生物沸石覆盖削减富营养化水体磷负荷是可行的.

\section{2 削减底泥间隙水中磷负荷能力}

实验历时 $121 \mathrm{~d}$, 对照和生物沸石覆盖系统表层 $\left(0 \sim 10 \mathrm{~cm}\right.$ ) 底泥间隙水中 $\mathrm{PO}_{4}^{3-}-\mathrm{P}$ 浓度都明显减小 (表 2 ), 原因是上覆水体水温的增加及低 DO 浓度促进表层底泥中磷释放 ( 图 2), 说明底泥间隙水中 $\mathrm{PO}_{4}^{3-}-\mathrm{P}$ 迁 移到上覆水体中, 从而增加上覆水磷负荷; 与对照系统相比, 生物沸石覆盖系统底泥间隙水 $\mathrm{PO}_{4}^{3-}-\mathrm{P}$ 浓度都有 一定程度的降低, 说明生物沸石覆盖对底泥间隙水有一定削减效果, 削减率为 $51.85 \% \sim 79.05 \%$, 平均值为 $59.80 \%$.

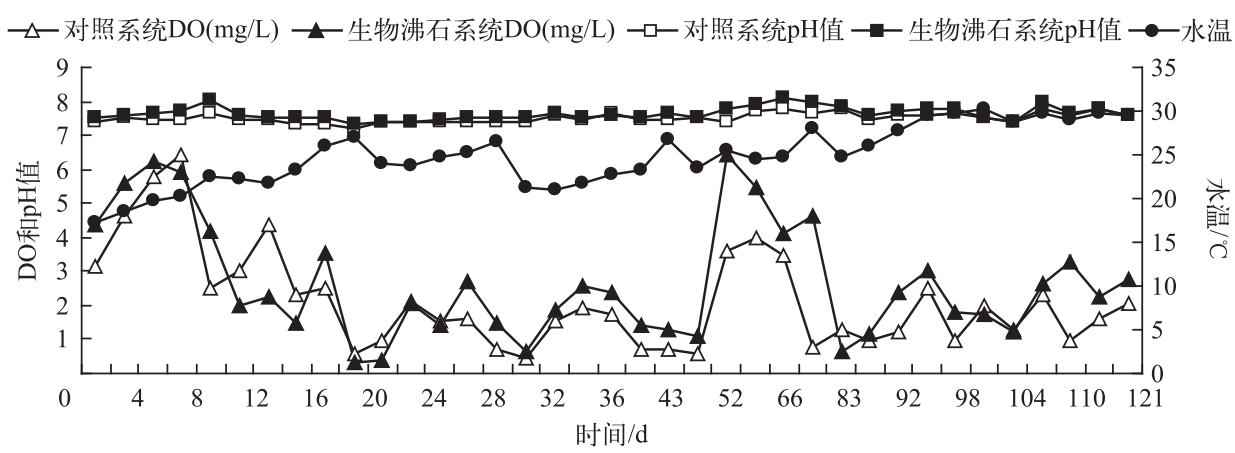

图 2 各系统上覆水中水温、DO 浓度和 $\mathrm{pH}$ 值的变化

Fig. 2 Changes of water temperature, dissolved oxygen concentration and $\mathrm{pH}$ of overlying water in two systems

\section{3 削减底泥中磷负荷能力}

对照系统底泥 TP、IP 和 OrP 含量均有不同程度减少 (表 3), 说明由于实验过程中上覆水体水温逐渐增 

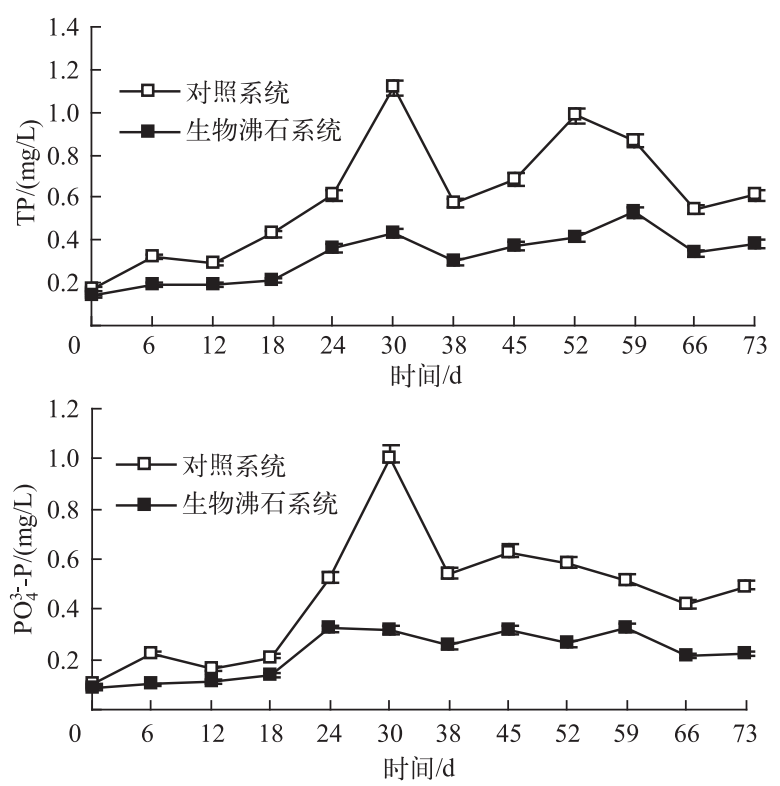

图 3 各系统上覆水中 $\mathrm{TP}$ 和 $\mathrm{PO}_{4}^{3-}-\mathrm{P}$ 浓度的变化

Fig. 3 Changes of TP and $\mathrm{PO}_{4}^{3-}-\mathrm{P}$ concentrations of overlying water in two systems

表 2 各系统底泥间隙水 $\mathrm{PO}_{4}^{3-}-\mathrm{P}$ 浓度垂向变化

Tab.2 Vertical changes of $\mathrm{PO}_{4}^{3-}-\mathrm{P}$ concentrations in interstitial water from two systems

\begin{tabular}{cccc}
\hline \multirow{2}{*}{$\begin{array}{c}\text { 底泥深度/ } \\
\mathrm{cm}\end{array}$} & $\begin{array}{c}\text { 实验前 } \\
\mathrm{PO}_{4}^{3-}-\mathrm{P} /(\mathrm{mg} / \mathrm{L})\end{array}$ & \multicolumn{2}{c}{ 实验后 $\mathrm{PO}_{4}^{3-}-\mathrm{P} /(\mathrm{mg} / \mathrm{L})$} \\
\cline { 3 - 4 } $0 \sim 3$ & $1.32 \pm 0.06$ & $0.77 \pm 0.04$ & $0.33 \pm 0.02$ \\
$3 \sim 6$ & $1.45 \pm 0.05$ & $0.66 \pm 0.03$ & $0.29 \pm 0.01$ \\
$6 \sim 10$ & $1.45 \pm 0.05$ & $1.05 \pm 0.04$ & $0.22 \pm 0.01$ \\
$10 \sim 20$ & $0.28 \pm 0.01$ & $0.27 \pm 0.01$ & $0.13 \pm 0.01$ \\
$20 \sim 50$ & $0.21 \pm 0.01$ & $0.51 \pm 0.02$ & $0.23 \pm 0.01$ \\
\hline
\end{tabular}

加促进底泥向上覆水体释放磷污染物, 增加上覆 水磷负荷. 与对照相比, 生物沸石覆盖系统对表 层 $(0 \sim 20 \mathrm{~cm})$ 底泥 TP 削减率为 7.64\% 13.49\%, 平均值为 $11.28 \%$; 对 IP 削减率为 $9.24 \%$ $13.99 \%$, 平均值为 $11.82 \%$; 对 $\mathrm{OrP}$ 削减率为 $6.91 \% \sim 14.81 \%$, 平均值为 $11.11 \%$, 说明生物沸 石覆盖对底泥磷负荷有一定削减效果.

对照系统表层 $(0 \sim 20 \mathrm{~cm})$ 底泥中 D-P、Al-P 和 Ca-P 垂向含量都有不同程度减少 (表 3 ), 而 Fe-P、RP 垂向含量有增加也有减少, 但总体有增 加趋势, 说明扬州古运河底泥的不同形态无机磷 之间转化比较复杂, 需要通过进一步研究底泥不

同形态无机磷转化机制. 与对照相比, 生物沸石覆盖系统对表层 $(0 \sim 20 \mathrm{~cm})$ 底泥中 D-P、Al-P、Fe-P 和 Ca-P 平均削减率分别为 $13.7 \% 、 15 \% 、 18.2 \%$ 和 $11.6 \%$, 而 RP 含量提高了 1 倍左右, 说明生物沸石覆盖能将底泥中 不稳定的无机磷 (D-P、Al-P 和 Fe-P) 转化为稳定状态的无机磷 (RP), 同时少部分的比较稳定的 Ca-P 也转 化为更为稳定的 RP, 因此, 生物沸石覆盖有利于将底泥中不稳定的无机磷 (DP、AL-P、Fe-P 和 Ca-P) 转化为 稳定的无机磷 (RP), 有助于磷的固定.

\section{4 削减磷负荷作用机理讨论}

1 ) 沸石对磷的物理吸附. 目前研究报道的沸石或改性沸石对磷吸附量大小差异性较大 ${ }^{[13,17-20]}$, 主要因 为沸石类型、初试溶液浓度、沸石投加量、沸石粒径等因素对沸石吸附量影响比较大,不同的实验条件下,得 到的沸石磷吸附量不同. 初始溶液磷浓度越高, 沸石对磷吸附量越大; 沸石投加量越多, 对溶液中磷削减效 果越好, 但单位质量沸石吸附磷量可能存在减少; 沸石粒径越小, 对磷吸附量越大, 但沸石粒径太小, 自身重 量太轻不利于覆盖. 因此, 在实际应用前, 有必要在应用对象现有的条件下进行实验研究, 估算出沸石对磷 吸附量、找到沸石最佳投加量及最佳粒径, 指导实际应用. 
表 3 各系统底泥中不同形态磷含量的垂向变化

Tab.3 Vertical changes of different phosphorus contents in sediments from two systems

\begin{tabular}{|c|c|c|c|c|c|c|c|}
\hline \multirow{2}{*}{ 磷形态 } & \multirow{2}{*}{ 时间 } & \multirow{2}{*}{ 系统 } & \multicolumn{5}{|c|}{ 底泥深度/cm } \\
\hline & & & $0 \sim 3$ & $3 \sim 6$ & $6 \sim 10$ & $10 \sim 20$ & $20 \sim 50$ \\
\hline \multirow[t]{3}{*}{$\mathrm{TP} /(\mathrm{mg} / \mathrm{kg})$} & 实验前 & 各系统 & $4330 \pm 420$ & $4481 \pm 360$ & $4481 \pm 450$ & $3690 \pm 424$ & $4092 \pm 356$ \\
\hline & 实验后 & 对照 & $3794 \pm 364$ & $3825 \pm 298$ & $3971 \pm 418$ & $3872 \pm 380$ & $3808 \pm 398$ \\
\hline & & 生物沸石 & $3504 \pm 278$ & $3305 \pm 269$ & $3546 \pm 408$ & $3362 \pm 340$ & $3634 \pm 288$ \\
\hline \multirow[t]{3}{*}{$\mathrm{IP} /(\mathrm{mg} / \mathrm{kg})$} & 实验前 & 各系统 & $840 \pm 78$ & $1047 \pm 98$ & $1047 \pm 116$ & $847 \pm 89$ & $962 \pm 75$ \\
\hline & 实验后 & 对照 & $840 \pm 68$ & $833 \pm 76$ & $905 \pm 102$ & $879 \pm 89$ & $828 \pm 106$ \\
\hline & & 生物沸石 & $754 \pm 66$ & $756 \pm 54$ & $780 \pm 46$ & $756 \pm 98$ & $828 \pm 80$ \\
\hline \multirow[t]{3}{*}{$\mathrm{OrP} /(\mathrm{mg} / \mathrm{kg})$} & 实验前 & 各系统 & $3490 \pm 320$ & $3434 \pm 280$ & $3434 \pm 290$ & $2843 \pm 296$ & $2843 \pm 284$ \\
\hline & 实验后 & 对照 & $2954 \pm 216$ & $2992 \pm 186$ & $3066 \pm 284$ & $2993 \pm 279$ & $2980 \pm 322$ \\
\hline & & 生物沸石 & $2750 \pm 128$ & $2549 \pm 168$ & $2766 \pm 270$ & $2606 \pm 166$ & $2806 \pm 264$ \\
\hline \multirow[t]{3}{*}{$\mathrm{DP} /(\mathrm{mg} / \mathrm{kg})$} & 实验前 & 各系统 & $9.61 \pm 0.88$ & $9.95 \pm 1.02$ & $9.95 \pm 0.78$ & $8.97 \pm 0.81$ & $10.94 \pm 0.68$ \\
\hline & 实验后 & 对照 & $6.43 \pm 0.45$ & $8.44 \pm 9.58$ & $7.94 \pm 0.74$ & $8.36 \pm 0.89$ & $8.04 \pm 0.92$ \\
\hline & & 生物沸石 & $5.56 \pm 0.50$ & $5.93 \pm 0.62$ & $6.03 \pm 0.46$ & $8.36 \pm 0.52$ & $7.70 \pm 0.84$ \\
\hline \multirow[t]{3}{*}{$\mathrm{Al}-\mathrm{P} /(\mathrm{mg} / \mathrm{kg})$} & 实验前 & 各系统 & $311 \pm 26$ & $423 \pm 28$ & $423 \pm 39$ & $332 \pm 38$ & $388 \pm 42$ \\
\hline & 实验后 & 对照 & $311 \pm 22$ & $319 \pm 26$ & $323 \pm 36$ & $343 \pm 38$ & $325 \pm 30$ \\
\hline & & 生物沸石 & $305 \pm 28$ & $291 \pm 26$ & $273 \pm 32$ & $283 \pm 18$ & $309 \pm 352$ \\
\hline \multirow[t]{3}{*}{$\mathrm{Fe}-\mathrm{P} /(\mathrm{mg} / \mathrm{kg})$} & 实验前 & 各系统 & $296 \pm 28$ & $382 \pm 28$ & $382 \pm 42$ & $287 \pm 31$ & $321 \pm 29$ \\
\hline & 实验后 & 对照 & $315 \pm 26$ & $296 \pm 19$ & $350 \pm 28$ & $291 \pm 25$ & $273 \pm 30$ \\
\hline & & 生物沸石 & $238 \pm 23$ & $250 \pm 18$ & $286 \pm 32$ & $252 \pm 26$ & $283 \pm 26$ \\
\hline \multirow[t]{3}{*}{$\mathrm{Ca}-\mathrm{P} /(\mathrm{mg} / \mathrm{kg})$} & 实验前 & 各系统 & $201 \pm 18$ & $206 \pm 14$ & $206 \pm 30$ & $191 \pm 22$ & $213 \pm 17$ \\
\hline & 实验后 & 对照 & $188 \pm 18$ & $186 \pm 14$ & $192 \pm 21$ & $207 \pm 28$ & $192 \pm 17$ \\
\hline & & 生物沸石 & $177 \pm 12$ & $183 \pm 24$ & $182 \pm 21$ & $170 \pm 20$ & $201 \pm 14$ \\
\hline \multirow[t]{3}{*}{$\mathrm{RP} /(\mathrm{mg} / \mathrm{kg})$} & 实验前 & 各系统 & $22 \pm 2$ & $26 \pm 3$ & $26 \pm 2$ & $29 \pm 3$ & $30 \pm 2$ \\
\hline & 实验后 & 对照 & $19 \pm 2$ & $23 \pm 1$ & $33 \pm 3$ & $29 \pm 2$ & $30 \pm 1$ \\
\hline & & 生物沸石 & $41 \pm 3$ & $49 \pm 2$ & $48 \pm 3$ & $58 \pm 4$ & $60 \pm 5$ \\
\hline
\end{tabular}

2) 沸石吸附水中 $\mathrm{NH}_{4}^{+}-\mathrm{N}$ 交换出来的 $\mathrm{Ca}^{2+} 、 \mathrm{Mg}^{2+}$ 等阳离子与底泥释放的 $\mathrm{PO}_{4}^{3-}$ 结合形成沉淀物固定在沸 石表面 ${ }^{[17]}$. 课题组前期研究表明 ${ }^{[13]}$, 在同等条件下, 沸石对含有 $\mathrm{NH}_{4}^{+}-\mathrm{N}$ 和 $\mathrm{PO}_{4}^{3-}-\mathrm{P}$ 溶液的磷去除率为 $22 \%$, 明显高于仅含有 $\mathrm{PO}_{4}^{3-}-\mathrm{P}$ 溶液的 $4 \%$, 说明由 $\mathrm{NH}_{4}^{+}-\mathrm{N}$ 交换出来的 $\mathrm{Ca}^{2+} 、 \mathrm{Mg}^{2+}$ 等阳离子与 $\mathrm{PO}_{4}^{3-}$ 结合形成沉淀物 这一作用途径对削减磷负荷贡献高于沸石对磷的物理吸附.

3) 生物沸石覆盖能将底泥中不稳定的无机磷 ( D-P、Fe-P 和 Al-P) 或少部分较稳定的无机磷 ( Ca-P) 转化 为稳定的无机磷 (RP). 其结论与其他研究结果趋于一致, 但不同研究条件下, 沸石、生物沸石、改性沸石覆 盖修复过程不同形态无机磷转化机制均存在些不同 ${ }^{[5,21-24]}$. Yin 等 ${ }^{[25]}$ 研究表明, 铝改性硅镁土覆盖能将 $\mathrm{P}_{\text {mobile }}$ (底泥可迁移磷) 转化为 Ca-P; Yang 等 ${ }^{[26]}$ 研究表明锆改性沸石覆盖能将 BD-P (碳酸氢钠提取磷) 转为 $\mathrm{NaOH}-\mathrm{P}$ (氢氧化钠可提取磷) 和 RP. 可见, 污染底泥原位覆盖修复过程不同形态无机磷转化机制非常复杂, 影响的因素很多,需要进一步研究.

\section{3 结论}

1) 生物沸石薄层覆盖能有效削减富营养化水体磷负荷, 覆盖强度为 $2 \mathrm{~kg} / \mathrm{m}^{2}$ 的生物沸石覆盖( 厚度约 2 $\mathrm{mm}$ ) 对上覆水中 $\mathrm{TP}$ 削减率 $57.41 \%$, 对上覆水中 $\mathrm{PO}_{4}^{3-}-\mathrm{P}$ 的削减率为 $60.03 \%$; 对底泥间隙水中 $\mathrm{PO}_{4}^{3-}-\mathrm{P}$ 的削 减率为 $59.80 \%$; 对表层底泥 $(0 \sim 20 \mathrm{~cm})$ 中 TP 削减率为 $11.28 \%$, 对 IP 削减率为 $11.82 \%$; 对 OrP 削减率为 $11.11 \%$,利用生物沸石薄层覆盖技术削减富营养化水体磷负荷是可行的.

2) 生物沸石薄层覆盖能将底泥中不稳定的无机磷 ( D-P、Fe-P 和 Al-P) 或少部分较稳定的无机磷 ( Ca-P) 
转化为稳定的无机磷 (RP), 说明生物沸石覆盖不仅能削减液相中磷负荷, 而且能将固相中不稳定的无机磷 转化为稳定的无机磷.

\section{4 参考文献}

[ 1 ] Lewis WM, Wurtsbaugh WA, Paerl HW. Rationale of control of anthropogenic nitrogen and phosphorus to reduce eutrophication of inland waters. Environmental Science and Technology, 2011, 45:10300-10305.

[ 2 ] Nilsson P, Jansson M. Hydrodynamic control of nitrogen and phosphorus turnover in an eutrophicated estuary in the Baltic. Water Research, 2002, 36: 4616-4626.

[ 3 ] Pan G, Dai LC, Li L et al. Reducing the recruitment of sedimented algae and nutrient release into the overlying water using modified soil/sand flocculation-capping in eutrophic lakes. Environmental Science and Technology, 2012, 46: $5077-5084$.

[ 4 ] Förstner U, Apit SE. Sediment remediation: U. S. focus on capping and monitored natural recovery. Fourth international conference on remediation of contaminated sediment. Journal of Soils and Sediments, 2007, 7: 351-358.

[ 5 ] Kim G, Jung W. Role of sand capping in phosphorus release from sediment. Journal of Civil Engineering, 2010, 14(6): 815-821.

[ 6 ] Li Dapeng, Huang Yong, Li Xiang. Phosphorus adsorption and immobility by sediments with ferric salt addition. Environmetal Chemistry, 2013, 32(5): 797-802(in Chinese with English abstract). [李大鹏, 黄勇, 李祥. 底泥加人铁盐对 水体磷的吸收和固定. 环境化学, 2013, 32(5): 797-802.]

[ 7 ] Shin EW, Han JS. Phosphate adsorption on aluminium-impregnated mesoporous silicates: Surface structure and behaviour of adsorbents. Environmental Science and Technology, 2004, 38: 912-917.

[ 8 ] Berg U, Neumann T, Donnert D et al. Sediment capping in eutrophic lakes-efficiency of undisturbed calcite barriers to immobilize phosphorus. Applied Geochemistry, 2004, 19(11) : 1759-1771.

[ 9 ] Lin JW, Zhan YH, Zhu ZL. Evaluation of sediment capping with active barrier system (ABS) using calcite/zeolite mixtures to simultaneously manage phosphorus and ammonium release. Science of the Total Environment, 2011, 409: 638-646.

[ 10] Huang TL, Zhou ZM, Xu JL et al. Biozeolite capping for reducing nitrogen load of the ancient in Yangzhou City. Water Science and Technology, 2012, 66 (2): 336-344.

[11] Özkundakci D, Hamilton DP, Gibbs MM. Hypolimnetic phosphorus and nitrogen dynamics in a small, eutrophic lake with a seasonally anoxic hypolimnion. Hydrobiologia, 2011, 661: 5-20.

[12] Meis S, Spears BM, Maberly SC et al. Sediment amendment with phoslock in Clatto reservoir (Dundee, UK) : Investigating changes in sediment elemental composition and phosphorus fractionation. Journal of Environmental Management, 2012, 93(1): 185-193.

[13] Zhou Zhenming. In situ remediation of contaminated sediment using combined biological and physicochemical technology in the urban rivers and lakes[Dissertation]. Xi' an: Xi' an University of Architecture \& Technology, 2013( in Chinese with English abstract). [ 周真明. 城市河湖污染底泥原位生物/物化组合修复技术研究 [ 学位论文]. 西安: 西安建筑科 技大学, 2013.]

[14] State Environmental Protection Administration ( SEPA) of China ed. Monitoring and analyzing methods of water and wastewater, 4th ed. Beijing: China Environmental Science Press, 2002(in Chinese). [ 国家环境保护总局. 水和废水监 测分析方法: 第 4 版. 北京: 中国环境科学出版社, 2002.]

[15] Jin Xingcan, Tu Qingying eds. Eutrophication investigation specification of lakes, 2th ed. Beijing: China Environmental Science Press, 1990( in Chinese). [ 金相灿, 屠清瑛. 湖泊富营养化调查规范: 第 2 版. 北京: 中国环境科学出版 社, 1990.]

[16] Ju Zewen, Wei Zhiqin, Dong Hong. Effects of aquatic vegetation rehabilitation on phosphorus in water and sediments of urban landscape waters. J Lake Sci, 2015, 27(2) : 234-242(in Chinese with English abstract). DOI 10.18307/2015.0206. [琚泽文, 蔚枝沁, 邓泓. 水生植被恢复对城市景观水体磷浓度及沉积物磷形态的影响. 湖泊科学, 2015, 27(2): 234-242.]

[17] Zhang Chuanguang, Zhang Naiming, Yu Xiufang. Effect of denitrification and dephosphorization of thermal modified clinoptilolite for eutrophic water. Chinese Journal of Environmental Engineering, 2013, 7(5) : 1665-1670( in Chinese with 
English abstract). [张传光, 张乃明, 于秀芳. 热改性斜发沸石对富营养化水体的脱氮除磷效果. 环境工程学报, 2013, 7(5): 1665-1670.]

[18] Duan Ning, Wu Yiyuan, Zhang Yinfeng. Adsorption kinetics and thermodynamics of diatomite/zeolite composite adsorbent nitrogen and phosphorus removal. Bulletin of the Chinese Ceramic Society, 2014, 33(12): 3151-3158 (in Chinese with English abstract). [段宁, 吴依远, 张银凤. 硅藻土/沸石复合颗粒吸附材料脱氮除磷的吸附动力学及热力学分析. 硅酸盐通报, 2014, 33(12): 3151-3158.]

[19] Yang Mengjuan, Lin Jianwei, Zhan Yanhui et al. Immobilization of phosphate in Taihu Lake sediment-water systems using aluminum-modified zeolites and zirconium-modified Zeolites as Amendments. Research of Environmental Sciences, 2014,27 (11)：1351-1359(in Chinese with English abstract). [杨孟娟, 林建伟, 詹艳慧等. 铝和锆改性沸石对太湖底泥-水 系统中溶解性磷酸盐的固定作用. 环境科学研究, 2014, 27(11) : 1351-1359.]

[20] Zhang Xiangling, Chen Junjie, Guo Lu et al. Analysis on the removal efficiency and mechanisms of phosphorus by modified zeolites substrates coated with ldhs reacted by different metal compounds in laboratory-scale vertical-flow constructed wetlands. Environmental Science, 2014, 35(12) : 4553-4559(in Chinese with English abstract). [张翔凌, 陈俊杰, 郭露 等. 垂直流人工湿地 LDHs 覆膜改性沸石基质强化除磷效果及其机制. 环境科学, 2014, 35( 12) : 4553-4559.]

[21] Akhurst D, Jones GB, McConchie DM. The application of sediment capping on phosphorous speciation and mobility in a sub-tropical dunal lake. Marine and Freshwater Research, 2004, 55: 715-725.

[22] Gibbs MM, Hickey CW, Özkundakci D. Sustainability assessment and comparison of efficacy of four P-inactivation agents for managing internal phosphorus loads in lakes: sediment incubations. Hydrobiologia, 2011, 658: 253-275.

[23] Xiong WH, Peng J. Laboratory-scale investigation of ferrihydrite-modified diatomite as a phosphorus co-precipitant. Water, Air and Soil Pollution, 2011, 215: 645-654.

[24] Sun Shiquan, Jiang Changbo, Zhao Gang et al. Influencing factors of zeolite in-situ remediation system to control moderately labile organic phosphorus migration and transformation in shallow water. China Environmental Science, 2015, 32(2) : 550-557 (in Chinese with English abstract). [孙土权, 蒋昌波, 赵刚等. 沸石覆盖原位控制湖泊内源中等活性有机磷 迁移转化. 中国环境科学, 2015, 32(2): 550-557.]

[25] Yin HB, Kong M. Reduction of sediment internal P-loading from eutrophic lakes using thermally modified calcium-rich attapulgite-based thin-layer cap. Journal of Environmental Management, 2015, 151: 178-185.

[26] Yang MJ, Lin JW, Zhan YH et al. Immobilization of phosphorus from water and sediment using zirconium-modified zeolites. Environmental Science and Pollution Research, 2015, 22 : 3606-3619. 